\title{
Surveillance programs watching for colistin-resistant infections
}

I

f the recently discovered MCR-1 gene led to colistin resistance in Canada, it would be pinpointed quickly, say leaders of Canada's surveillance programs for antimicrobial resistance.

First found in China last November, MCR-1 confers colistin resistance in bacteria such as Escherichia coli. Its presence has now been confirmed in 11 countries, including Canada. MCR-1 has been found in two patients who returned from foreign destinations and in two meat samples in Ontario, said Michael Mulvey, chief of antimicrobial resistance and nosocomial infections at the National Microbiology Laboratory and coauthor of a forthcoming letter to Lancet Infectious Diseases detailing the discoveries.

Colistin, an antibiotic developed in the 1950s, is used as the "last drug of defence" against gram-negative bacterial infections resistant to other antibiotics, said Mulvey.

"It is an important drug in the arsenal," agreed George Zhanel, the director of the Canadian Antimicrobial Resistance Alliance. "It would be a disaster if we could not use it."

To date, surveillance programs have found very little colistin-resistant bacteria in Canada. Zhanel said hospital samples collected through the alliance's Canadian Ward Surveillance Study (CANWARD), which began in 2007, show only a "handful" of colistin-resistant $E$. coli samples. CANWARD has found that colistin resistance is higher in some other organisms, but some bacteria are inherently resistant.

At the same time, the Public Health Agency of Canada's (PHAC) Canadian Nosocomial Infection Surveillance Program (CNISP) has been monitoring resistance to carbapenem-class antibiotics in the Enterobacteriaceae family of gram-negative bacteria, including $E$. coli, since 2010 and has found that about $5 \%$ of samples have been colistin-resistant, said Mulvey.

The two surveillance programs have differing but complementary approaches, said Zhanel and Mulvey, and they often cooperate. CANWARD samples large numbers of pathogens found in 15 tertiary hospitals weighted to represent pop- ulation levels. Samples come from many common types of infections in blood, urine, wounds and the respiratory tract. They are tested for resistance against a comprehensive panel of antibiotics, including those in the development pipeline. The large number of samples allows statistical analysis on local, provincial and national levels.

"It allows us to do some elegant genetic studies such as those for MCR-1 and methicillin-resistant Staphylococcus aureus," said Zhanel.

Zhanel says that if MCR-1-based colistin resistance became a clinical problem, "CANWARD would pick that up for sure."

CNISP conducts surveillance in 54 sentinel hospitals, said Mulvey. Whereas CANWARD looks at all types of pathogens, CNISP targets five. It is part of broader animal and human antimicrobial surveillance within PHAC, including a sister program called the Canadian Integrated Program for Antimicrobial Resistance Surveillance, which provided the meat samples for the MCR-1 study. It goes "from farm to fork to flush," said Mulvey.

PHAC has combined the data from several programs that monitor antimicrobial use and resistance in a new Canadian Antimicrobial Resistance Surveillance System, which publishes an annual analysis report; the first appeared in March 2015. Mulvey described the system as "the first of its kind. It is a onestop shop to integrate the data coming out and summarize it at a higher level."

Zhanel and Mulvey emphasize the role of communication in surveillance for antimicrobial resistance. The National Microbiology Laboratory communicates with laboratories and hospitals across Canada as well as with its international counterparts, Mulvey said. "Part of our role as a national lab is looking at what's happening elsewhere in the world and communicating with other national labs in the world, to be prepared when something arises." - Carolyn Brown, Ottawa, Ont.

CMAJ 2016. DOI:10.1503/cmaj.109-5242 